\title{
Realization of an Efficient Optical Interconnect Using L-Shape Photonic Waveguide via Finite Difference Time Domain Method
}

\section{Sangram Kumar Nayak}

Gandhi Institute of Engineering and Technology

Pratap Kumar Panigrahi

Gandhi Institute of Engineering and Technology

Gopinath Palai ( $\sim$ gpalai28@gmail.com )

Gandhi Institute For Technological Advancement https://orcid.org/0000-0002-9891-493X

\section{Research Article}

Keywords: LED, Photodiode, 2-D crystal waveguide, FDTD

Posted Date: February 16th, 2022

DOI: https://doi.org/10.21203/rs.3.rs-1347264/v1

License: (c) (i) This work is licensed under a Creative Commons Attribution 4.0 International License.

Read Full License 


\title{
Realization of an efficient optical interconnect using L-Shape photonic waveguide via finite difference time domain method
}

\author{
Sangram Kumar Nayak ${ }^{1}$, Pratap Kumar Panigrahi ${ }^{1}$, G.Palai $^{*}$ \\ 1. Department of Electrical and Electronics Engineering, GIET University, Gunupur, \\ 2. Department of Electronics and communication Engineering, Gandhi Institute for Technological \\ Advancement, Bhubaneswar, India (gpalai28@gmail.com, gpalai_ece@gita.edu.in)
}

\begin{abstract}
The present research focuses on two dimensional L-shape waveguide for the realisation of optical interconnect at the signal of $1550 \mathrm{~nm}$, which will help for the transformation of signals from one chip to another. In this article, graphene based light emitting diode generates the wavelength of $1550 \mathrm{~nm}$, where PIN photodiode converts the optical signal (wavelength $1550 \mathrm{~nm}$ ) to its electrical counterpart. The proposed wave guide (11×11 silicon rods) with $\mathrm{L}$ shape defect allows the signal from source to detector. The principle of mechanism relies on the propagation of field along with the defect path which is made through finite difference time domain method. Moreover, there is no loss associated with the structure during the transformation of signals. The computational result infers that the efficiency of LED is about $99.98 \%$ where the efficiency of photodiode is $99.94 \%$. Finally, the suggested silicon based waveguide acts as a good candidate for the realisation of an efficient optical interconnect.
\end{abstract}

Keywords:

LED, Photodiode, 2-D crystal waveguide, FDTD

\section{Introduction}

Research on optical interconnect has been focused time to time to minimize the losses in the photonic devices. The optical interconnect plays vital role to realise an efficient photonic integrated circuit. Basically, it controls the losses as well as efficiency of the light wave circuits. Interconnects is a backbone of the planar light wave circuit because losses associated with different devices are directly depends on the connector. Maximum amount of losses take place at interconnects as well as in the coupling regions. Photonic devices could be realised by the controlling of coupling loss and waveguide parameters, so that the signal coming from source (Light emitting diode) would reflect at the destination (photo detector) without any loss in the inter-medium stage. Since photonic based interconnects play an important role for designing an efficient device, many works relating to the same have done with the help of different types of photonic crystal waveguides [1-10]. For example; optical printed circuit board is addressed in the reference [1], where authors focuses on planar graded index waveguide along with the fabrication techniques. In reference [2] , a data centre network $(\mathrm{DCN})$ is analyzed to realise a photonic interconnect through the principle of wavelength division demuliplexing where nanoscale switching is envisaged. Similarly different types of optical interconnects are discussed in the reference [3] for data centers networks. Moreover this works 
claimed that the proposed DCN would provide simplicity for routing, less energy consumption, good scalability, lack of packet loss and etc. Also, reference [4] focusing on the high energy efficiency, large bandwidth and low cost optical inter connect which will be suitable for current photonic integrated circuits. Again, reference [5] reviews on Photonic Integrated Networked Energy (PINE) efficient data center to study the architecture of optical interconnects, where the present PINE can be using for high performace computing. Reference [6] discloses the construction of optical memory along with the random access memory which acts as excellent optical inter connect. An ultra-high speed optical interconnects with record single lane based on conventional silicon photonic modulator with Mach-Zehnder structure is discussed in the reference [7]. Similarly, monolithic integration of electro-optic modulators based on the Pockels effect in barium titanate (BTO) thin films is addressed in the reference [8] where pockels modulator acts as a good optical interconnect and suitable for photonic integrated circuits. Furthermore, reference [9] and [10] focuses on the one dimensional optical interconnects using defect and defect less polymer based one dimensional photonic crystal structure.

Though, above said works have been explained by different authors, the present article discusses an L-shape two dimensional photonic structure waveguide through which zero loss could be realised. The present device has another advantage pertaining to the efficiency of LED, photo diode and coupling loss, which is less than $1 \%$. The proposed device could be used as optical interconnect as well as for chip to chip communication purposes. Keeping on importance of important of such device, the present paper organises as follows;

The introduction of the works is discussed in the section 1. The proposed structure and its operational mechanism are indicated in the section 2 . Similarly section 3 discusses the numerical computation along with the explanation to realise optical interconnect. Similarly section 4 presents the result and interpretation of the research. Finally, conclusion is mentioned in the section 5.

\section{Proposed structures}

The photonic crystal waveguide which is considered to realise an efficient optical interconnect, shown in figure 1(a). 


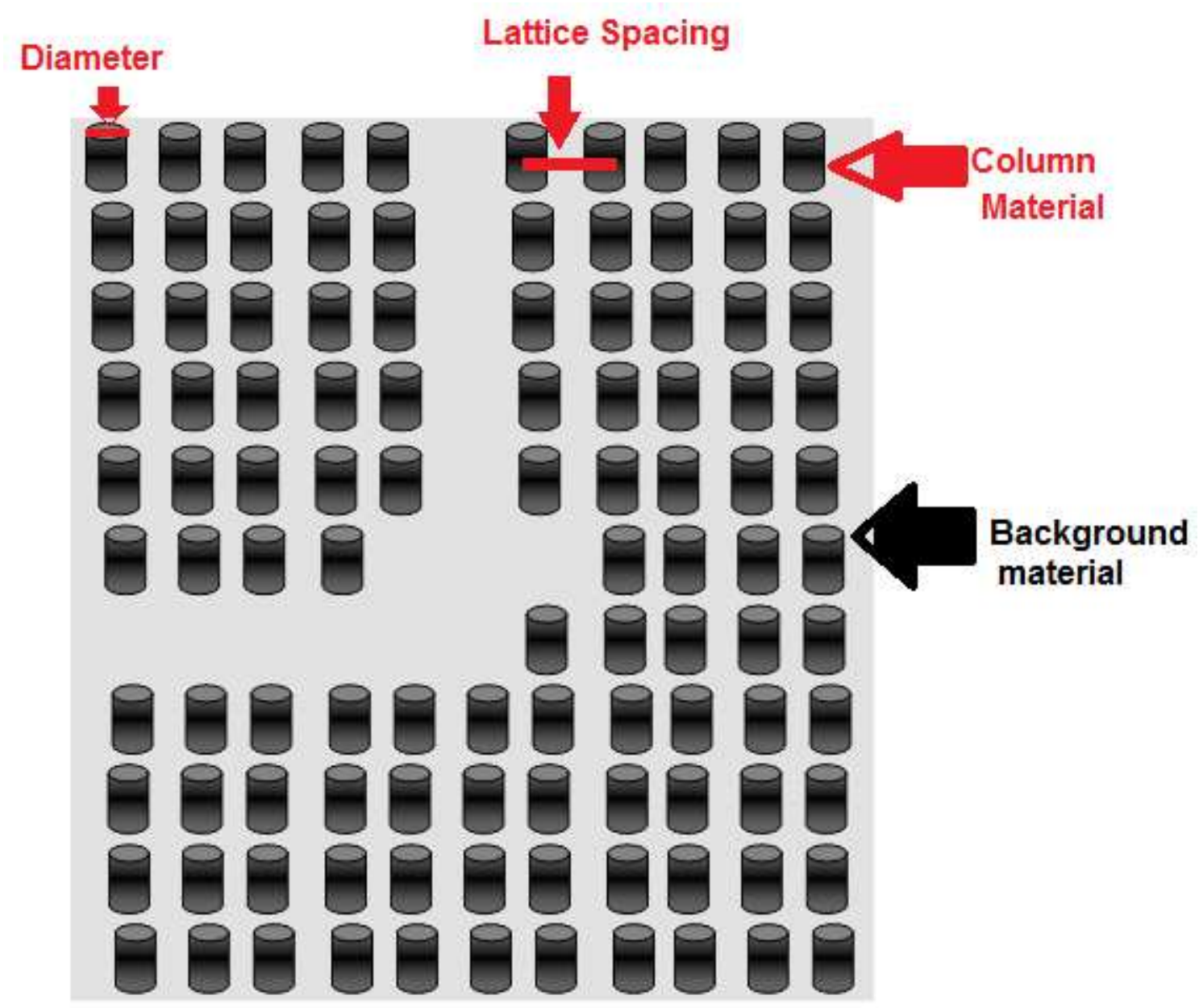

Figure 1(a) schematic of L-shape two dimensional photonic crystal waveguide

In this case, the structure consists of $11 \times 11$ silicon rods with air act as background material. Here $11 \times 11$ silicon rod is arranged such way that a series of silicon rods have been removed to fix $\mathrm{L}$ shape. The $\mathrm{L}$ shape is call as defect (absence of silicon rod) in the photonic waveguide. The simulation diagram corresponding to wavelength structure is shown in the 1(b) 


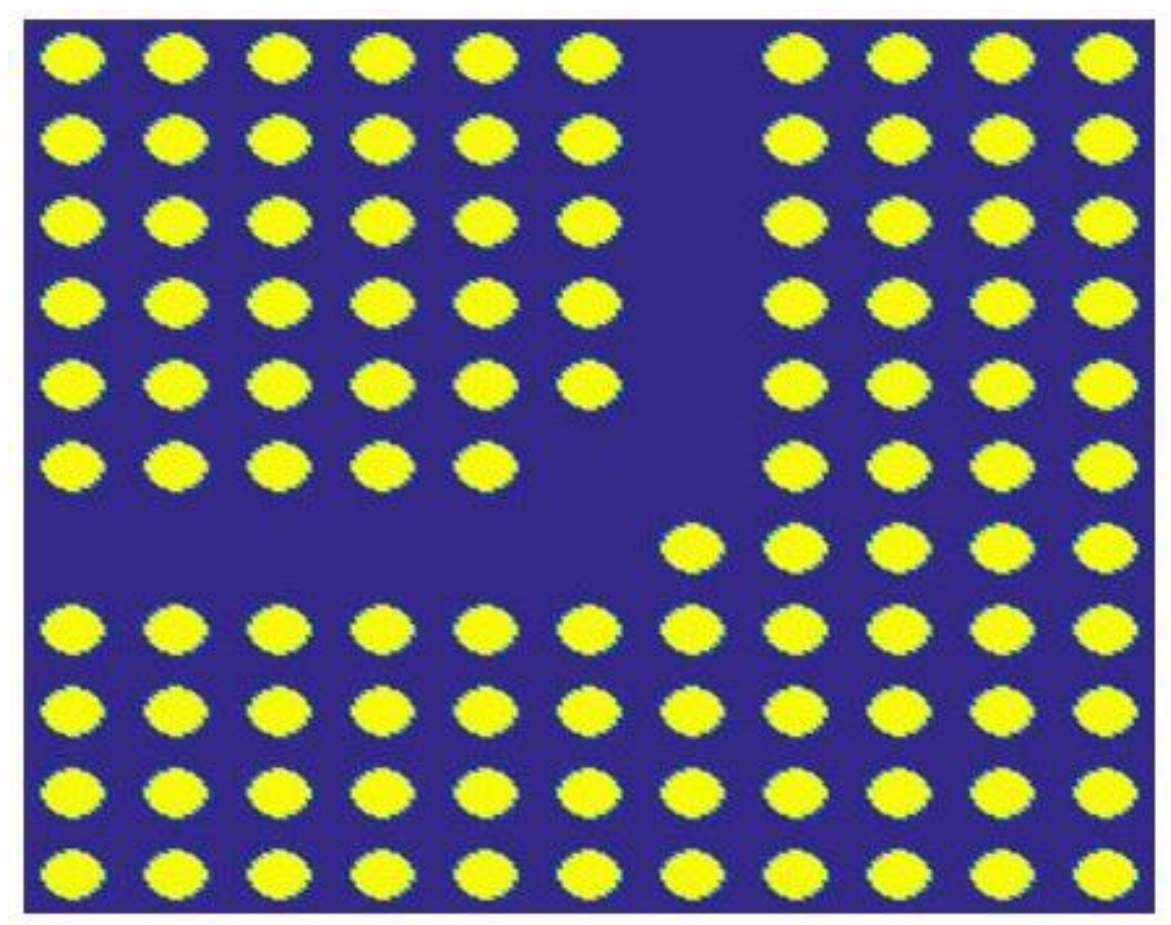

Figure 1(b); simulated figure for lattice spacing, $1 \mu \mathrm{m}$ and radius, $300 \mathrm{~nm}$

In this figure blue colour is represented as background material, where yellow colour is nothing but circular rod which is made up silicon rod, which are air material in side L-shape (defect). As far as configuration of same structure are concerned, we have chosen various type of radius of rods and lattice spacing of structure, which is taken of $1 \mu \mathrm{m}$. We did the simulation for finding the electric distribution for the entire radius with the help of finite difference time domain method. Further, efficient interconnectors have been confirmed from such simulation, which is discussed in the result and interpretation section. Moreover, considering the operational mechanism of photonic device, Figure 1(c) provides information regarding the transportation of signal from transmitter to receiver end through optical interconnect. 


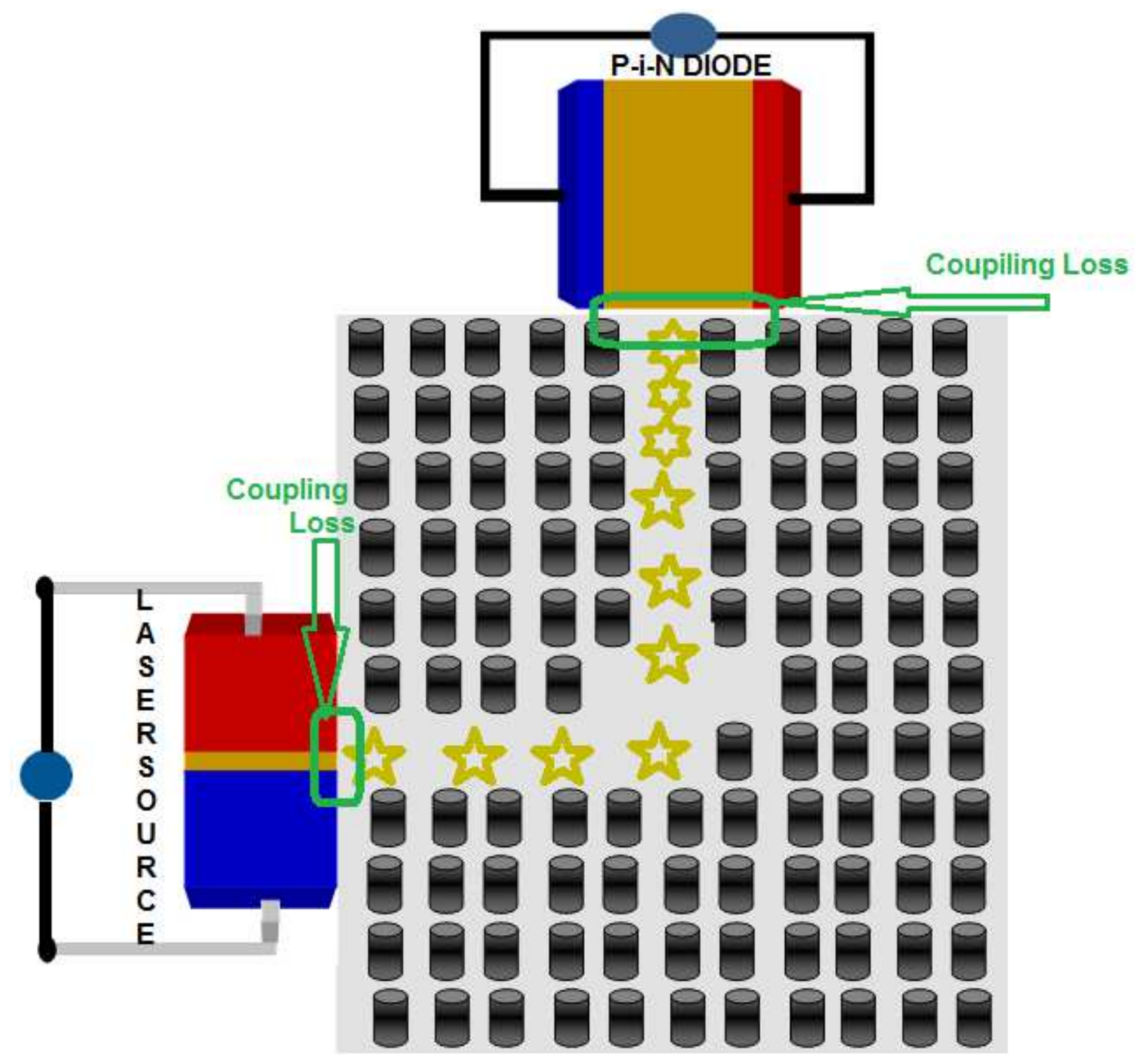

Figure 1(c); Mechanism of transformation of signals from transmitter to receiver

Figure 1(c) depicts the complete information for transportation of signal from transmitter end (LASER source) to receiver end ( $\mathrm{p}$-i-n photodiode) through L-shape bending waveguide. In this case we have seen that electrical signal in terms of volts incidents ( forward bias) to the light emitting diode then LED diode is designed in such way that it converts to optical counterpart, whose wavelength of $1.55 \mu \mathrm{m}$. The reason for choosing such signal is that $1550 \mathrm{~nm}$ belongs to the third optical communication windows which are frequently used in the recent communication system. After generating wavelength of $1.55 \mu \mathrm{m}$ from the light emitting diode (Laser Source), it passes through the silicon based waveguide. The waveguide is proposed in such way that no loss is found in the L shape path. Finally, it arrives at destination, i.e. $1.55 \mu \mathrm{m}$ reaches the photo detector. Further, photo detector is chosen in such way that it will convert optical signal to its electrical counterpart and finally signals appears in terms of electrical wave. To sum up it is inferred that whatever electrical signal is coming to transmitter end reaches at the output end.

Keeping the importance of different components for an operational mechanism from figure 1(c), figure 2 discusses various components, which are included in the photonic devices. 


\subsection{LED (Laser Source)}

The proposed light emitting diode is made up of GaAs based semiconductor where active layer is made up of multilayer of graphene. The same structure is shown in the figure 2(a).

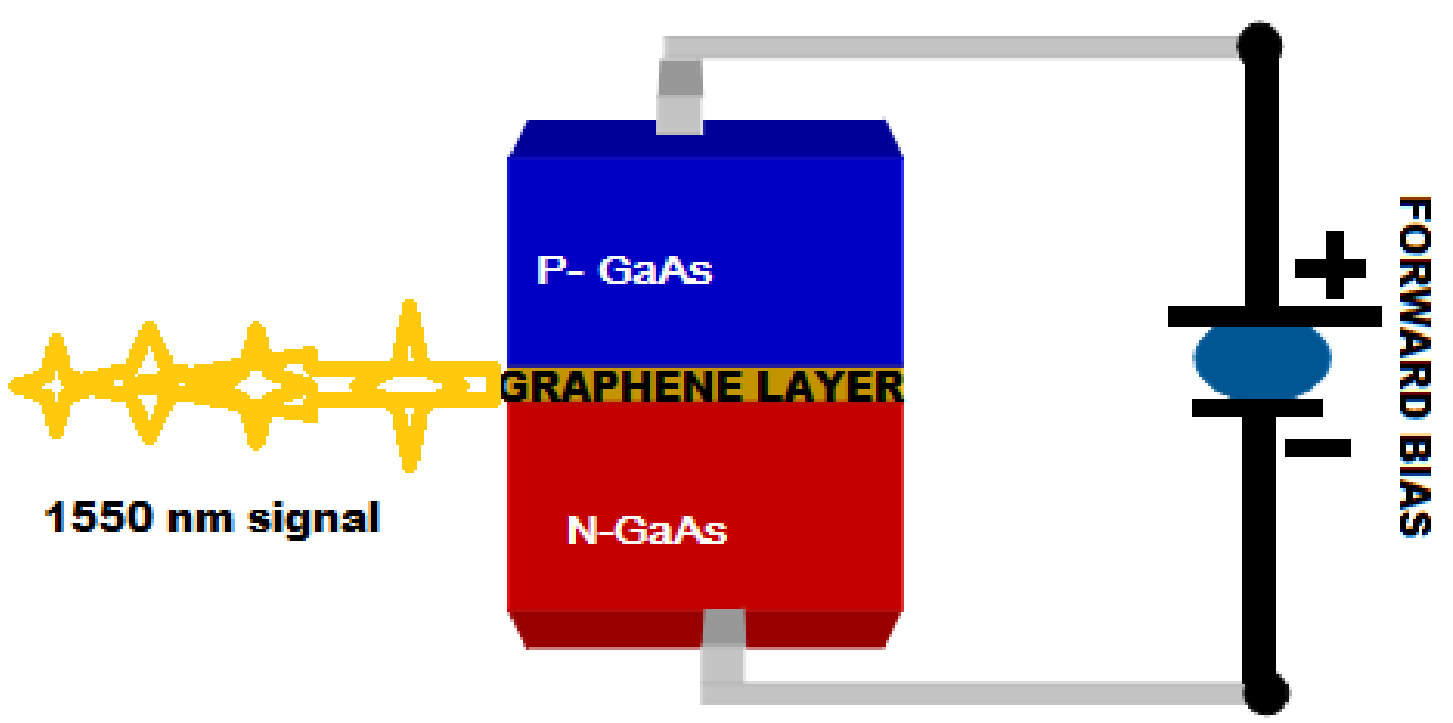

Figure 2(a), schematic diagram light emitting source

In this case $\mathrm{P}-\mathrm{GaAs}$ and $\mathrm{N}-\mathrm{GaAs}$ is taken as p-type and n-type region, where 9 layer of graphene (multilayer) is placed in the middle region, which is acting as active layer. As far as working mechanism is concerned, when potential (forward bias) is applied to the GaAs based LED then it will generate a signal of $1550 \mathrm{~nm}$ which is acting as monochromatic LASER beam. The reason for considering $1550 \mathrm{~nm}$ is that this signal is suitable for optical communication.

\subsection{Photonic waveguide}

In this structure (figure 1), L-shape waveguide is taken as air material which will allow $100 \%$. (Without making any loss) signal at a particular configuration. This waveguide will solve the issues relating the bending loss. Moreover, such photonic waveguide can be implanted for chip to chip communication in the integrated circuit.

\subsection{Photonic diode}




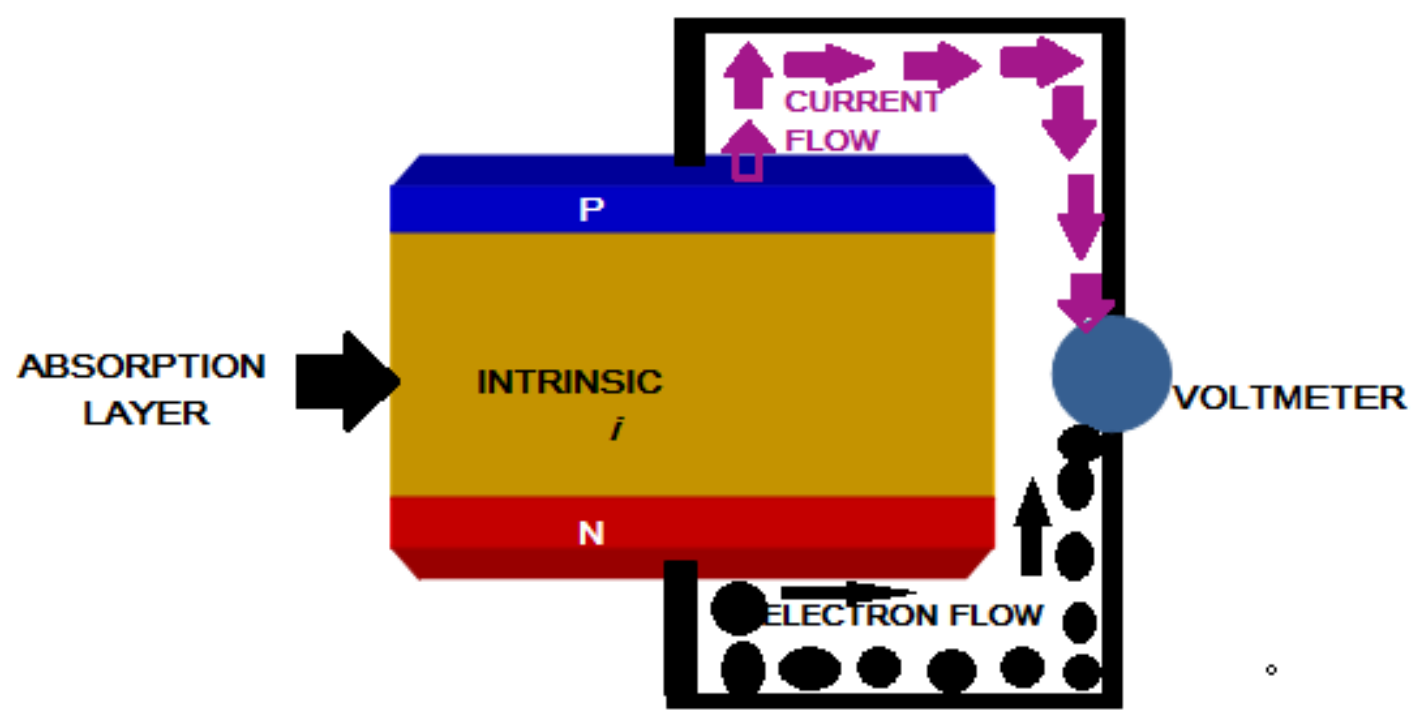

2(b) Schematic diagram of P-i-n photodiode

Figure 2(b) shows the schematic structure of P-i-N photo diode, which is the silicon based p-type, n-type and intrinsic layer, which plays vital role. For example; the size of the intrinsic layer is high as compared to the $p$ and $\mathrm{n}$ side. Because, the more amount of radiation absorb in an intrinsic region leads to enough amount of electron and hole pairs that give rise to the more amount of electric current . Here the thickness of intrinsic layer is taken of $10 \mu \mathrm{m}$, which is large as compared to $\mathrm{p}$ and $\mathrm{n}$ side (500 $\mathrm{nm}$ each). Apart from this, the proposed P-i-N photo diode provides high efficiency as well as good responsivity.

\section{Mathematical formulae}

The mathematics is used in this research is divided into several sections. For example; the computational efficiency of LED, the coupling efficiency/loss between LED and waveguide, efficiency of photonic crystal waveguide, the field distribution in L-shape region, coupling loss between waveguide and photodiode, efficiency and rensoponsivity of photodiode

\subsection{Efficiency of LED}

The efficiency of LED can be computed through internal and external quantum efficiency.

The expression for internal efficiency can be written as [11]

$$
\eta_{\text {int }}=\frac{K_{R}}{K_{R}+K_{n R}} \quad 1(a)
$$

Where $K_{R}$ and $K_{n R}$ are the radiative and non-radiative constant

Similarly the external efficiency can be written as [12]

$$
\eta_{\text {ext }}=\frac{1}{n_{a}\left(n_{a}+1\right)^{2}}
$$

Where $\eta_{\mathrm{a}}$ be the refractive index of an active layer

So the overall quantum efficiency of laser source can be expressed

$$
\eta_{\text {ext }} \eta=\eta_{\text {int }} \times \eta_{\text {ext }} \quad 1(\mathrm{c})
$$

\subsection{Coupling efficiency of LED and photonic crystal waveguide}

The coupling efficiency is defined as the ratio of power entering to the waveguide to the total power generating by the light emitting diode which can be written as [13] 


$$
\eta=\frac{\mathrm{W}_{\mathrm{L}}}{\mathrm{W}_{\mathrm{A}}}\left({\sqrt{n_{1}^{2}-n_{2}^{2}}}^{2}\right.
$$

where $\mathrm{W}_{\mathrm{L}}$ and $\mathrm{W}_{\mathrm{A}}$ is thickness of active layer of laser source and L-shape of the waveguide. Similarly $\eta_{1}$ and $\eta_{2}$ is the refractive index of waveguide and source respectively.

\subsection{Electric field distribution}

As far as the mathematical formulation pertaining to photonic crystal waveguide is concerned, basically plane wave expansion, transfer matrix method, beam propagation method and finite difference time domain method etc. are being frequently used now a days to study the photonic properties of photonic crystal structure. Finite difference time domain ( FDTD) is general technique for computing the propagation of electromagnetic waves in above said structure of random geometry. The FDTD is directly involved with discretization of Maxwell's equation and it is independent of propagation of direction and as a result of which, it deals with an accurate result with respect to experimental one. In this case time evolution of electromagnetic field distribution is cogitated in overall special domain. This leads to FDTD techniques is suitable for simulating different properties of the photonic crystal structure. To discretise the field distribution in the photonic crystal structure, Let us formulate the Maxwell's equations, which is given as [14-15]

$$
\begin{aligned}
& \nabla \times E=-\mu \frac{\partial H}{\partial t} \\
& \nabla \times H=-\varepsilon \frac{\partial E}{\partial t}
\end{aligned}
$$

Further explaining the said equation, expression for TMz mode can be expressed in Maxwell equation as

$$
\begin{aligned}
& \frac{\partial \mathrm{Ez}}{\partial \mathrm{t}}=\frac{1}{\varepsilon} \frac{\partial \mathrm{Hy}}{\partial \mathrm{x}}-\frac{\partial \mathrm{Hx}}{\partial \mathrm{y}} \\
& \frac{\partial \mathrm{Hx}}{\partial \mathrm{t}}=-\frac{1}{\mu_{0}}\left(\frac{\partial \mathrm{Hz}}{\partial \mathrm{y}}\right) \\
& \frac{\partial \mathrm{Hy}}{\partial \mathrm{t}}=\frac{1}{\mu_{0}}\left(\frac{\partial \mathrm{Ez}}{\partial \mathrm{x}}\right)
\end{aligned}
$$

Again considering Yee mesh, around the point $(i, j)$ and considering

$(\mathrm{i}, \mathrm{j})=(\mathrm{i} \Delta \mathrm{x}, \mathrm{j} \Delta \mathrm{y})$, the function $\mathrm{f}^{\mathrm{n}}(\mathrm{i}, \mathrm{j})=\mathrm{f}(\mathrm{i} \Delta \mathrm{x}, \mathrm{j} \Delta \mathrm{y}, \mathrm{n} \Delta \mathrm{t})$

The final set of equation can be defined as

$$
\begin{aligned}
& E_{z}{ }^{n+1}(i, j) \\
& =E_{z}{ }^{n}(i, j) \\
& +\frac{\Delta t c}{\varepsilon(i, j)}\left\{\frac{H_{y}{ }^{n+\frac{1}{2}}\left(i+\frac{1}{2}, j\right)-H_{y}{ }^{n+\frac{1}{2}}\left(i-\frac{1}{2}, j\right)-H_{x}{ }^{n+\frac{1}{2}}\left(i, j+\frac{1}{2}\right)-H_{x}{ }^{n+\frac{1}{2}}\left(i, j-\frac{1}{2}\right)}{\Delta y}\right\} \\
& \quad H_{x}{ }^{n+\frac{1}{2}}\left(i, j+\frac{1}{2}\right)=H_{x}{ }^{n-\frac{1}{2}}\left(i, j+\frac{1}{2}\right)-\frac{\Delta t}{\Delta y} c\left[E_{z}{ }^{n}(i, j+1)-E_{z}{ }^{n}(i, j)\right]
\end{aligned}
$$

Using equation 4, and employing $\mathrm{ABC}$ (absorbing boundary condition), the modal field of propagation can be determined, which is reflected in next section. 


\subsection{Coupling efficiency waveguide and photo diode}

The coupling efficiency between waveguide and photodiode can be defined as the ratio between the amounts of power entering to the photodiode to the amount of power emerging from the waveguide. Mathematically, it can be written as [16]

$$
\eta_{\text {abs }}=e^{-\alpha_{\text {coupling } \times L}}
$$

Where $\alpha_{\text {coupling. } L}$ is the absorption coefficient of index of photodiode and $\mathrm{L}$ be the thickness of the absorption layer of the p-i-n diode.

\subsection{Efficiency and responsitivity of photo diode}

The quantum efficiency of photodiode can be defined as the number of electron - hole pair generation per the total amount of photons incident on it.

Mathematically, it can be defined as

$$
\eta_{\mathrm{PD}}=\frac{\text { No of e-p pairs produced }}{\text { No.of Incident photons }} \times 100(\%) \quad 10(\mathrm{a})
$$

Basically, the efficiency of photo diode depends on the three layer structure parameter such as p, intrinsic and n-type semiconductor layer. In this case we have considered silicon based intrinsic layer and its efficiency can be written as [17]

$$
\eta_{P D}=(1-R)^{2} e^{-\left(\beta_{1} t_{1}+\beta_{2} t_{2}+\beta_{3} t_{3}\right)} 10(\mathrm{~b})
$$

Where $\mathrm{R}$ is called as reflection, $\beta 1, \beta 2$ and $\beta 3$ represents as absorption co efficient of $\mathrm{p}$, intrinsic and $\mathrm{n}$ type material respectively. Similarly, $\mathrm{t}_{1}, \mathrm{t}_{2}$ and $\mathrm{t}_{3}$ are the thickness of $\mathrm{p}$, intrinsic and $\mathrm{n}$-type semiconductor material, respectively. The result of the above efficiency can be done through plane wave expansion method.

Further, moving to obtain responsitivity of photodiode. It can be expressed as [18]

$$
R=\frac{\eta \lambda}{h c} \quad 10(\mathrm{c})
$$

Where, $\eta, \mathrm{h}$ and $\mathrm{c}$ are called as efficiency of the photodiode, planks constant and speed of light in vacuum. Here operating wavelength $(\lambda)$ is taken of $1550 \mathrm{~nm}$.

\section{Result and Interpretation}

The outcome of the research works provides an efficient optical interconnects devices for chip to chip communication. However the optical inter connector is restricted with $90^{\circ}$ bending loss or L type interconnects. The entire output relies on the different numerical treatment, whose supporting mathematical expressions are discussed in the previous sections. Considering the expression explained in the section 3 are indicated as follows.

\subsection{Efficiency of LED}

The equation 1(a) provides an expression for internal quantum efficiency where the equation 1(b) gives the expression for external quantum efficiency for laser source (Light Emitting Diode). Basically, the internal quantum efficiency depends on the radiative and non-radiative factor. Since we have considered GaAs semiconductor material which is direct band gap material and in this case, there is no non- radiative constant. So putting the vales of $\mathrm{K}_{\mathrm{nr}}$ is zero, the internal quantum efficiency is 1 which indicates $100 \%$ of internal quantum efficiency. Further, it is realised that three layer structure of $\mathrm{p}$ type GaAs, graphane layer and $\mathrm{n}$ type GaAs play a vital role. When signal come from chip 1 and reached at the LED then it converts to its light counterpart and the relation between them is 


$$
\lambda=\frac{h c}{e V}
$$

Moreover focussing on the above three layers, the transmittance (percentage) of generated signal by the optical source depends (overall quantum efficiency) on the equation 1(c) which relies on the equation (external quantum efficiency) 1(b) because internal quantum efficiency is $1(100 \%)$. The output of result is determined by using the equation $1(\mathrm{~b})$ and it is indicated in the figure $3(\mathrm{a})$.

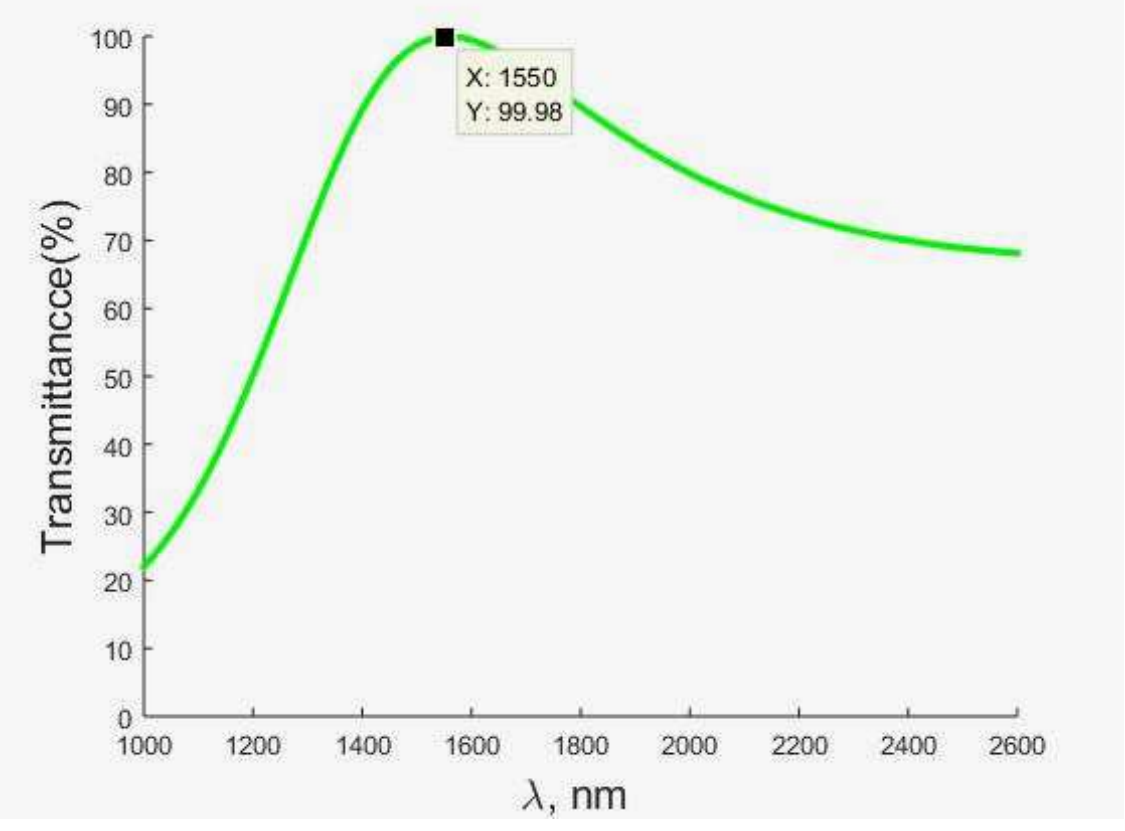

Figure 3, the transmittance (efficiency) of the laser source

In this graph 3, transmittance of signal by the LED structure is taken along vertical axis where wavelength of generated signal is taken along the horizontal axis. It is found that transmittance of signal gradually increases with respect to the wavelength and reaches at the peak value, then it starts to decrease pertaining to the increase of wavelength. Moreover, this also state that the efficiency of laser source is about $99.19 \%$ for generating the signal of $1550 \mathrm{~nm}$.

\subsection{Coupling efficiency between LED and photonic crystal waveguide}

From equation 1(d), it is realised that the coupling efficiency depends on the thickness of the active layer (multilayer of graphene) and waveguide length (L-shape). Apart from this, it is also found that it depends on the numerical aperture, which relies on the refractive index of active layer and proposed silicon based photonic waveguide with air as background material. Since the thickness of active layer $(530 \mathrm{~nm})$ is less than thickness $(1000 \mathrm{~nm})$ of L-shape (defective path light propagation through it), there is no possibility of loss between waveguide and source. Moreover, putting the values of refractive index of photonic crystal waveguide, it is found that the coupling efficiency is about 1 . It indicates that there is no coupling loss associated with the layer between LED (light source) and photonic waveguide.

\subsection{Transportation of signal in waveguide}

The principle of transportation of signal depends on the electric field distribution in the waveguide which is made using the equation (4) with the help of finite difference time domain method. To carry out the same, we have considered various type of configuration. For example; radius of silicon rod is varied from $0.01 \mu \mathrm{m}$ to $0.49 \mu \mathrm{m}$. However, the lattice spacing of the structure is $1 \mu \mathrm{m}$. The simulation result corresponding to each 
combination of lattice spacing and radius of the column cylinder. Though simulation for different combination of lattice spacing and radius of silicon rods , $(0.01 \mu \mathrm{m}, 1 \mu \mathrm{m}),(0.05 \mu \mathrm{m}, 1 \mu \mathrm{m}), 0.1 \mu \mathrm{m}, 1 \mu \mathrm{m}),(0.15 \mu \mathrm{m}, 1$ $\mu \mathrm{m}),(0.2 \mu \mathrm{m}, 1 \mu \mathrm{m}),(0.25 \mu \mathrm{m}, 1 \mu \mathrm{m}),(0.3 \mu \mathrm{m}, 1 \mu \mathrm{m}),(0.35 \mu \mathrm{m}, 1 \mu \mathrm{m}),(0.4 \mu \mathrm{m}, 1 \mu \mathrm{m}),(0.45 \mu \mathrm{m}, 1 \mu \mathrm{m})$, and $(0.49 \mu \mathrm{m}, 1 \mu \mathrm{m})$, have been done, the simulation structure and output result from $(0.01 \mu \mathrm{m}, 1 \mu \mathrm{m}),(0.4$ $\mu \mathrm{m}, 1 \mu \mathrm{m}), .(0.49 \mu \mathrm{m}, 1 \mu \mathrm{m})$, is shown in figure $4(\mathrm{a}, \mathrm{b}), 5(\mathrm{a}, \mathrm{b})$ and $6(\mathrm{a}, \mathrm{b})$ respectively.

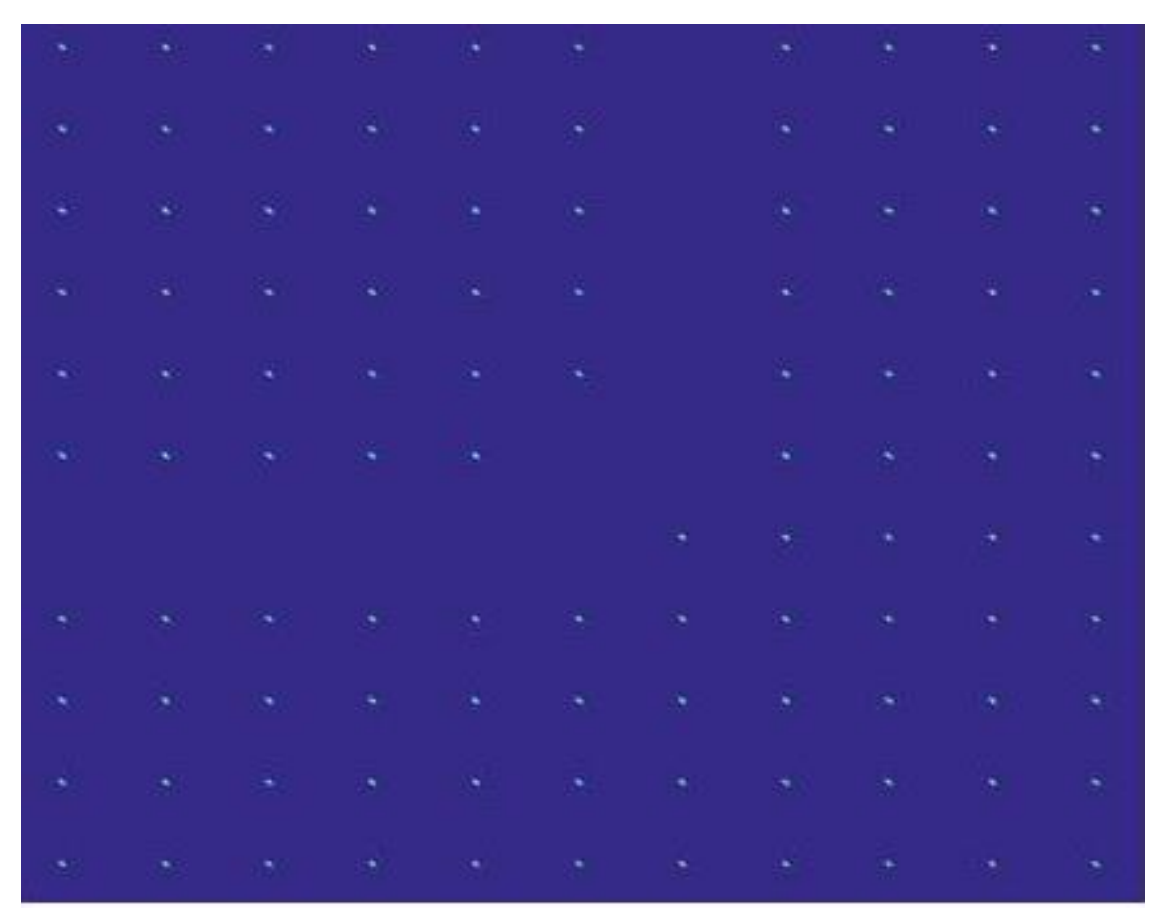

Figure 4(a); simulated 2D photonic crystal waveguide for radius of column , $0.01 \mu \mathrm{m}$

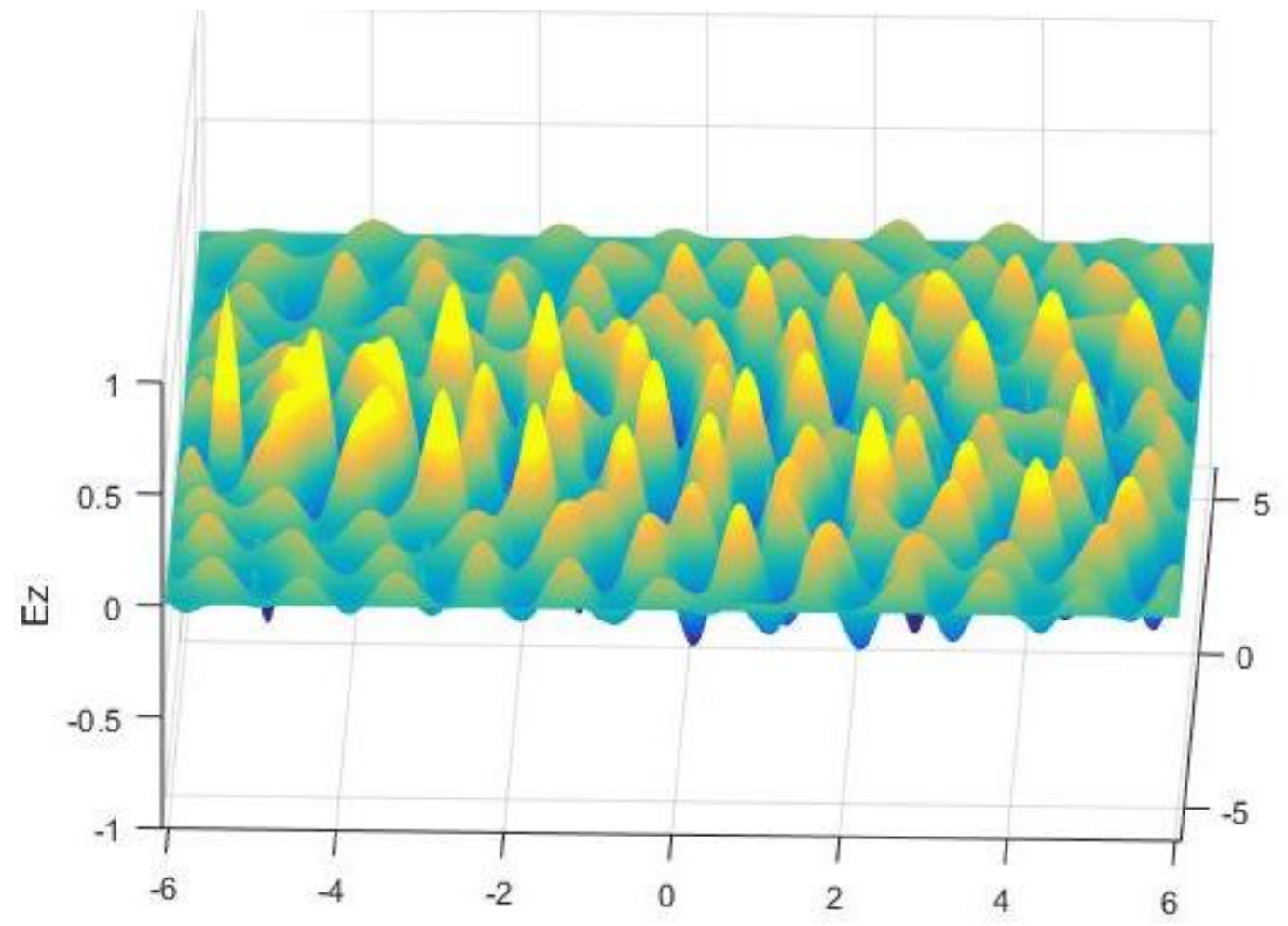

Figure 4(b); Electricfield in 2D photonic crystal waveguide for radius of column, $0.01 \mu \mathrm{m}$ 


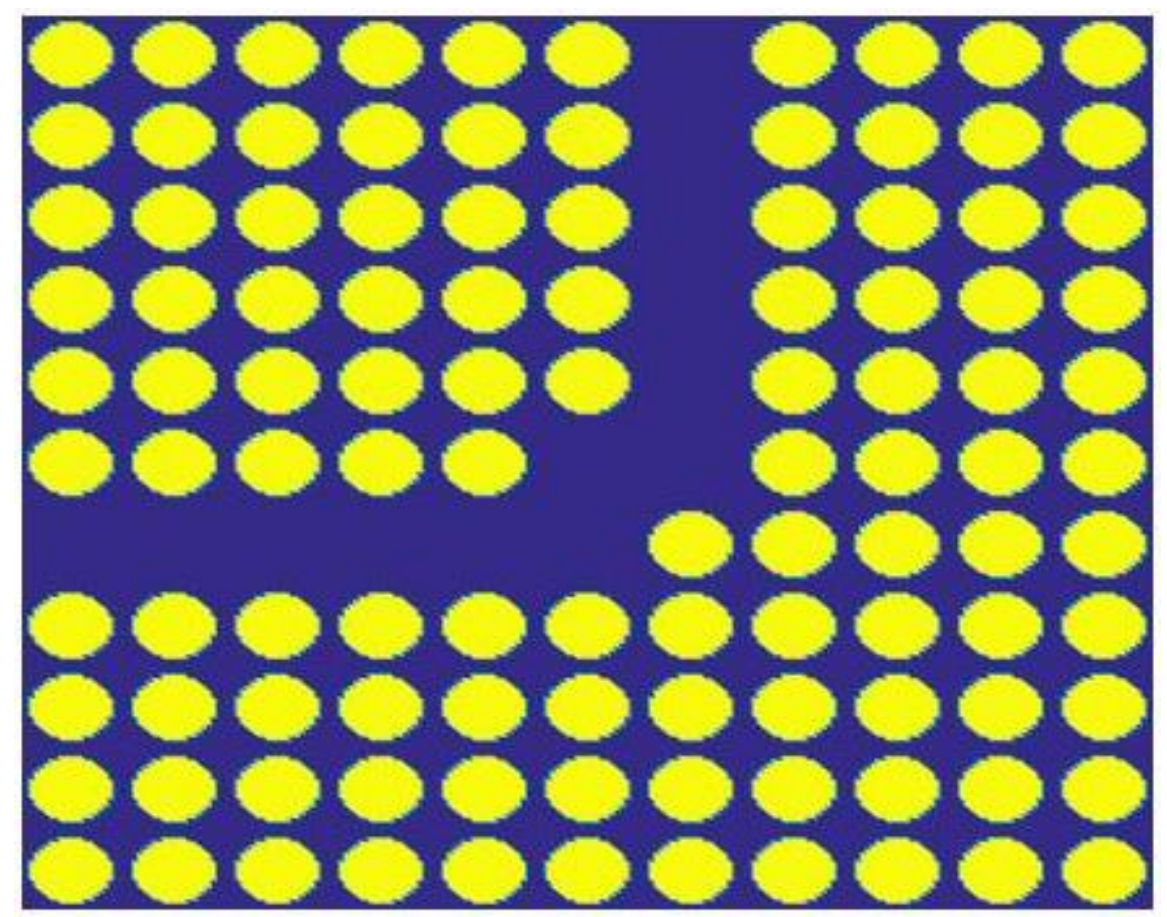

Figure 5(a); simulated 2D photonic crystal waveguide for radius of column , $0.4 \mu \mathrm{m}$

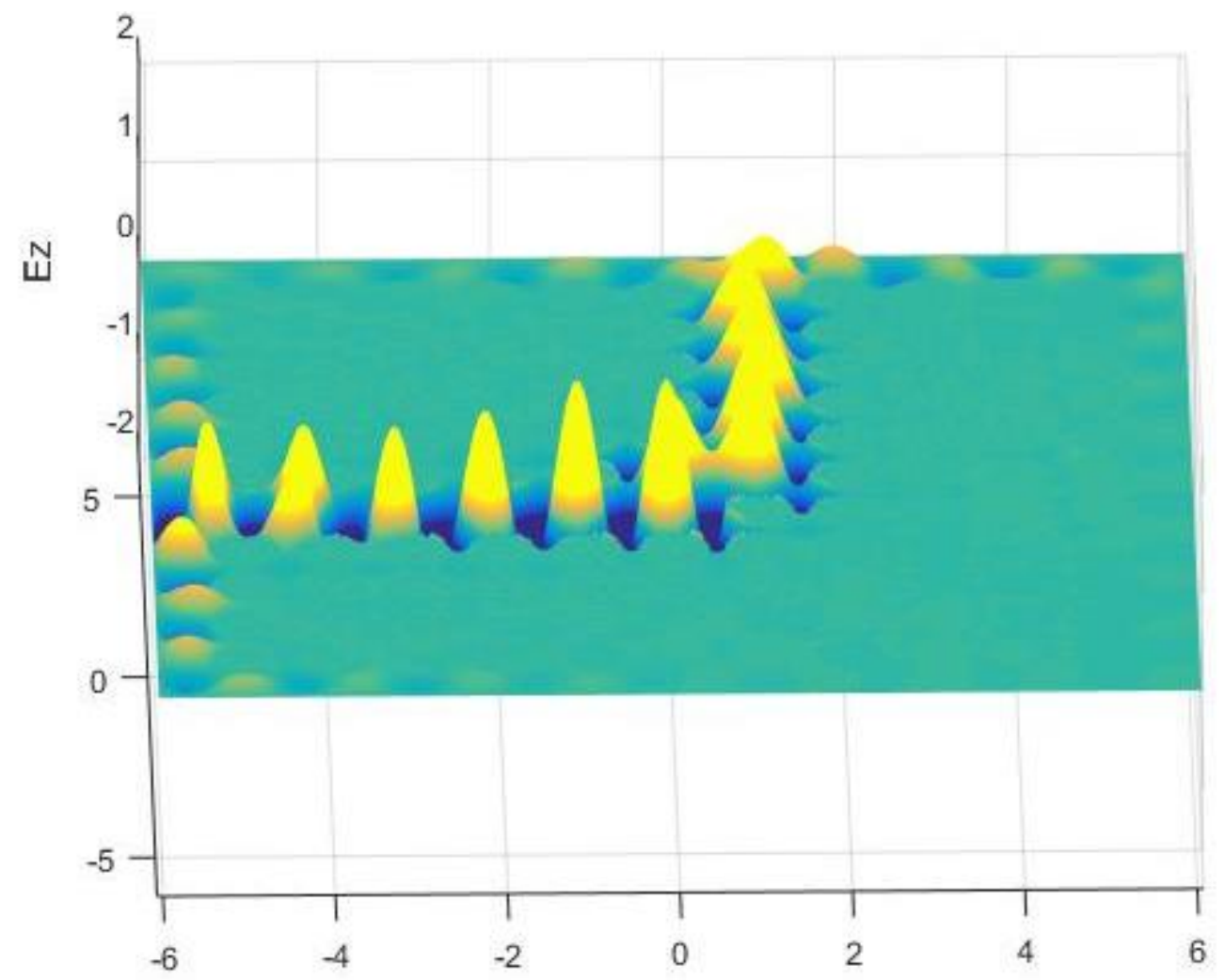

Figure 5(b); Electric field distribution in 2D photonic crystal waveguide for radius of column, $0.4 \mu \mathrm{m}$ 


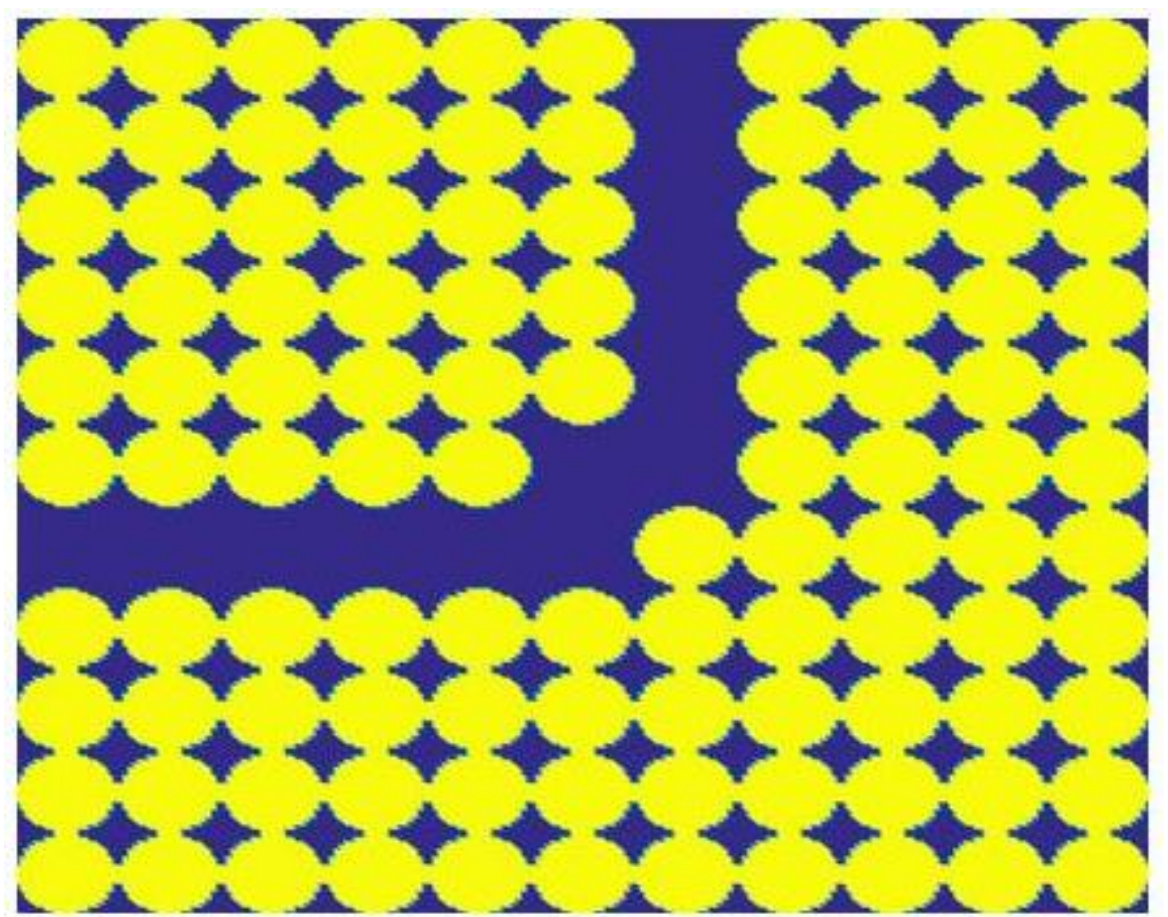

Figure 6(a); simulated 2D photonic crystal waveguide for radius of column , $0.49 \mu \mathrm{m}$

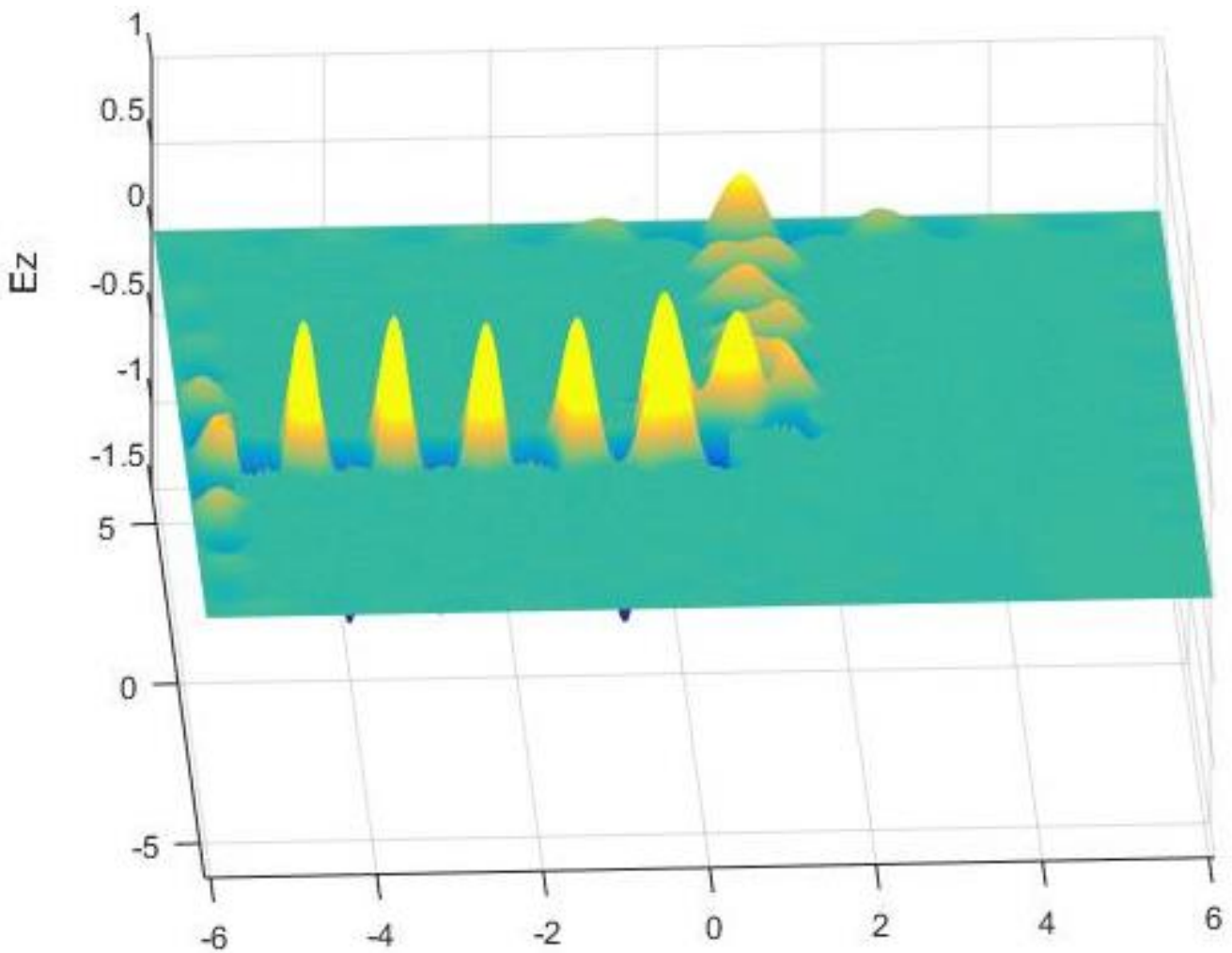

Figure 6(b); Electricfield distribution in 2D photonic crystal waveguide for radius of column $.0 .49 \mu \mathrm{m}$ 
Figure 4(a), 5(a) and 6(a) represent the simulation result of schematic of defect based two dimensional photonic crystal waveguide, whose consisting of $11 \times 11$ silicon rods with air treat as background material. In this case , L-shape line defect is made where signal can propagate through it. Considering these structure it is observed that the radius of silicon rods are $0.01 \mu \mathrm{m}, 0.4 \mu \mathrm{m}$ and $0.49 \mu \mathrm{m}$ corresponding to the figure 4(a), 5(a) and 6(a) respectively. Further, the simulation results for electric field distribution corresponding to each structure are shown in the figures 4(b), 5(b) and 6(b) respectively. In case figure 5(b), it observed that the signal is scattered in the crystal waveguide and it does not follow the path of L-shape path. So it will not be suitable to solve the bending loss issues. However, it is found from the figure 5(b) and 6(b) that no scattering loss is associated with it. It indicates that whatever signal coming from transmitter, it will arrive at the output end of the photonic waveguide. So it is inferred that, structure for configuration, $(0.4 \mu \mathrm{m}, 1 \mu \mathrm{m})$ and. $(0.49 \mu \mathrm{m}$, $1 \mu \mathrm{m})$ are suitable for solving bending loss issues. Hence structure corresponding to such material can be a good candidate for optical interconnect. Apart from this, similar principle indicates that structure having the radius from $0.3 \mu \mathrm{m}$ to $0.49 \mu \mathrm{m}$ and lattice spacing of $1 \mu \mathrm{m}$ could be an appropriate structure for reducing bending loss problem in the integrated circuits. The probable reason for such interesting result same is due to effective refractive index. For example the effective refractive index is more, then the speed of the signal in the waveguide would less which leads to the less scattering .Otherwise, if the effective refractive index is less, and then the speed of signal shall more which leads to the more scattering loss.

\subsection{Coupling efficiency between waveguide and photodiode}

The coupling efficiency is the ratio of amount of power entering to the photo diode to the amount of power emerging from the photo crystal waveguide. In this case, photonic waveguide $(1 \mu \mathrm{m})$ is less than the thickness $(10 \mu \mathrm{m})$ of absorption layer photodiode. Considering the equation (9), it is realised that the efficiency of photodiode depends on the coupling coefficient between waveguide structure and photodiode. Similarly, (P-i$\mathrm{N})$ coupling coefficient is zero at the signal of $1550 \mathrm{~nm}$, which is found from the literature[19]. So putting the value of coefficient, the coupling efficiency is 1 , which infers that coupling loss is zero.

\subsection{Efficiency and Responsivity of photodiode}

The efficiency of p-i-n photodiode depends on the number of electron generated with respect to the number of photon produced at intrinsic layer. Moreover it depends on the reflectance as well as absorbance, which is governed by equation $10(\mathrm{a}-\mathrm{b})$. Since it is three layer structure, the reflectance is determined using plane wave expansion method where absorption coefficient $\left(\beta_{1} \beta_{2}, \beta_{3}\right)$ at proposed thickness and operating wavelength is found to be zero [20]. Since the absorbance loss is zero in the photodiode, the efficiency depends on the reflectance only, which is carried out with the help of plane wave expansion method. The output efficiency in terms of transmittance pertaining to the wavelength $1550 \mathrm{~nm}$ is plotted in the figure 7 (a). 


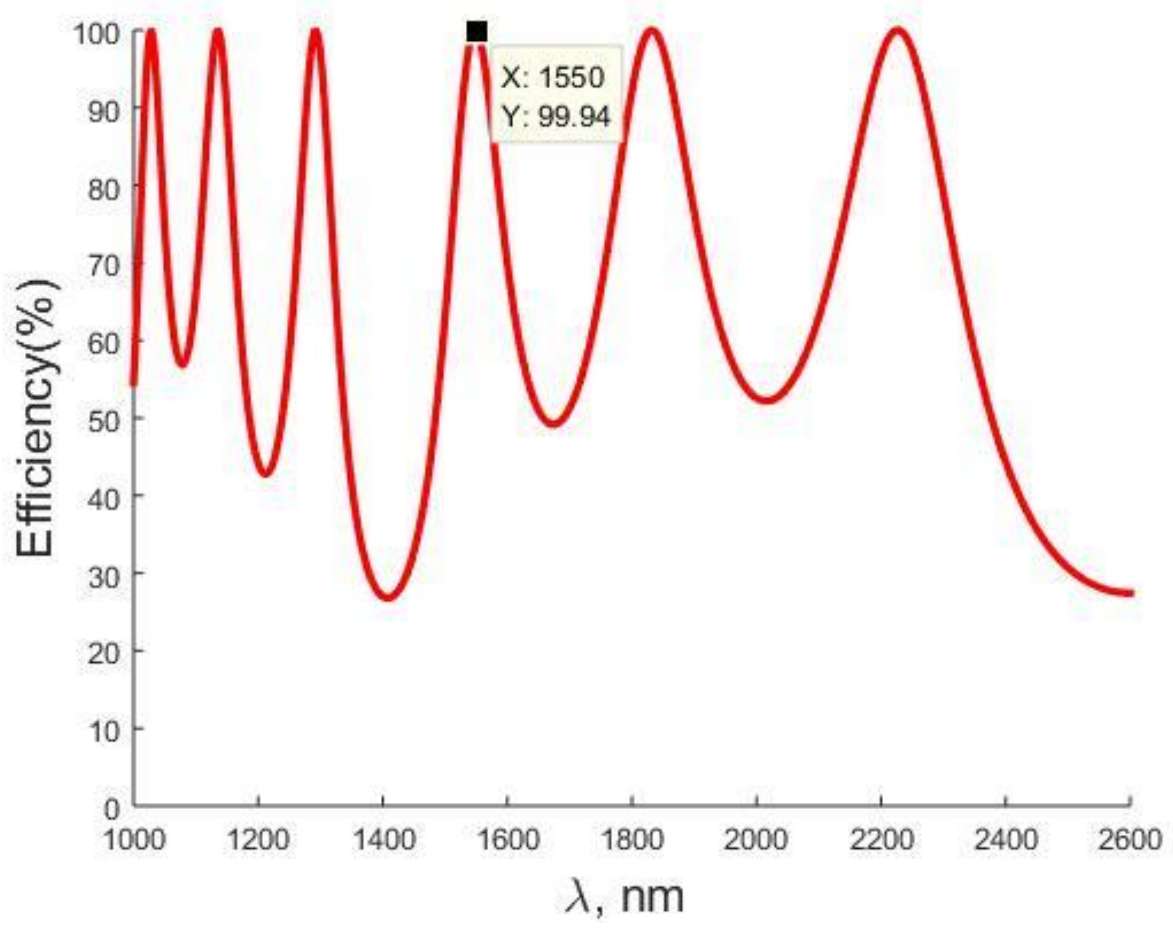

Figure 7; the output efficiency of p-i-n photo diode

In this figure; wavelength in $\mathrm{nm}$ and transmittance (\%) is taken along horizontal and vertical axis respectively. Here transmittance varies non-linearly with respect to the wavelength. The efficiency (transmittance) is found to be $99.94 \%$ at signal of $1550 \mathrm{~nm}$.

Further analysing the rensponsivity of the photodiode with the help of equation (11), it is found that resnponsivity depends on efficiency of structure and wavelength of input signal. Putting these values it is found that 1.25 , which are excellent outcomes with respect to current research scenario.

To sum up, it is realised that laser source shows an efficiency of $99.98 \%$, zero coupling loss between source and waveguide, zero loss photonic waveguide, no coupling loss between waveguide and photodiode, and $99.94 \%$ efficiency of photo diode with high resnponsivity could be a suitable optical interconnect for the $90^{\circ}$ bending loss, which could be robust element in photonic integrated circuit.

\section{Conclusion}

Silicon based two dimensional photonic crystal waveguide is proposed in this research to realise an efficient optical interconnect at the signals of $1550 \mathrm{~nm}$. The suitable combination of graphene based Laser diode, silicon based waveguide and p-i-n photo diode eradicates the bending loss issues. Moreover the output result indicates that coupling efficiency is more than $99 \%$,which could be a leading interconnect for integrated circuit. 


\section{References}

1. Competitive Evaluation of Planar Embedded Glass and Polymer Waveguides in Data Center Environments ;Richard Pitwon, Kai Wang, Akira Yamauchi, Takaaki Ishigure, Henning Schröder, Marcel Neitz, Mayank Singh, Appl. Sci. 2017, 7(9), 940; https://doi.org/10.3390/app7090940 - 13 Sep 2017

2. Nanoseconds photonic integrated switches for optical data center interconnect systems ; N. Calabretta; Kristif Prifti; Xuwei Xue; Fulong Yan; Bitao Pan; Xiaotao Guo ; Proc. SPIE 11286, Optical Interconnects XX, 1128605 (28 February 2020); doi: 10.1117/12.2550101

3. PCOI: Packet Classification-Based Optical Interconnect for Data Centre Networks; Rab Nawaz Jadoon, Mohsin Fayyaz, WuYang Zhou, Muhammad Amir Khan, Ghulam Mujtaba, Volume 2020 |Article ID 2903157 | https://doi.org/10.1155/2020/2903157

4. D.Tsiokos ,G.T.Kanellos, 2 - Optical interconnects: Fundamentals, Optical Interconnects for Data Centers; Woodhead Publishing Series in Electronic and Optical Materials; 2017, Pages 43-73;

5. Madeleine Glick, Nathan C. Abrams, Qixiang Cheng, Min Yee Teh,Yu-Han Hung, Oscar Jimenez, Songtao Liu, Yoshitomo Okawachi, Xiang Meng, Leif Johansson, Manya Ghobadi, Larry Dennison, George Michelogiannakis, John Shalf, Alan Liu, John Bowers, Alex Gaeta,Michal Lipson, Keren Bergman; PINE: Photonic Integrated Networked Energy efficient datacenters; Journal of Optical Communications and Networking; Vol. 12, No. 12 / December 2020 , Pages 443-456

6. Theoni Alexoudi, George Theodore Kanellos ,Nikos Pleros; Optical RAM and integrated optical memories: a survey; Light: Science \& Applicationsvolume 9, article number: 91 (2020)

7. Fan Zhang, Yixiao Zhu, Fan Yang, Lei Zhang, Xiaoke Ruan, Yanping Li, and Zhangyuan Chen ; Up to single lane 200G optical interconnects with silicon photonic modulator ; Optical Fiber Communication Conference Postdeadline Papers 2019; (Optical Society of America, 2019),paper Th4A.6; https://doi.org/10.1364/OFC.2019.Th4A.6,

8. Felix Eltes, Christian Mai, Daniele Caimi, Marcel Kroh, Youri Popoff, Georg Winzer, Despoina Petousi, Stefan Lischke, J. Elliott Ortmann, Lukas Czornomaz, Lars Zimmermann, Jean Fompeyrine, Stefan Abel; A BaTiO3-based electro-optic Pockels modulator monolithically integrated on an advanced silicon photonics platform; Journal of light wave technology,

9. G.Palai , S.K.Tripathy, DeoPrakas, K.D.Verma, High efficiency polymer grating SOI structures for optical interconnect: An application of organic photonics; Optik; Volume 127, Issue 22, November 2016, Pages 10948-10952

10. G. Palai, T. K. Dhir, B. Nath ,S. L. Patra ; Modelling overall transmitted efficiency at $1550 \mathrm{~nm}$ for polymer grating Silicon-on-insulator structure with defect; Frontiers of Optoelectronics volume 6, pages 153-159 (2013) 
11. Jong-In Shim, Dong-Soo Shin; Measuring the internal quantum efficiency of light-emitting diodes: towards accurate and reliable room-temperature characterization; Nanophotonics 2018; 7(10): 16011615 .

12.M.Z.Farah Khaleda,B.Vengadaesvaran,N.A. Rahim; Chapter 18 - Spectral response and quantum efficiency evaluation of solar cells: a review; Energy Materials: Fundamentals to Applications; 2021, Pages 525-566.

13. Eun-Hyun Park; Moon-Jung Kim; Young-Se Kwon; Microlens for efficient coupling between LED and optical fiber; IEEE Photonics Technology Letter; 11(14), 1987; 439 - 441

14. A. Taflove (1980). "Application of the finite-difference time-domain method to sinusoidal steady state electromagnetic penetration problems" (PDF). IEEE Trans. Electromagn. Compat. 22 (3): 191-202.

15. CS Mishra, Anand Nayyar, Sandeep Kumar, Bandana Mahapatra, G Palai; FDTD approach to photonic based angular waveguide for wide range of sensing application; Optik,176(1), 56-59,2019

16. Donghwan Ahn, Ching-yin Hong, Lionel C. Kimerling, and Jurgen Michel; Coupling efficiency of monolithic, waveguide-integrated Si photodetectors; Applied Physics Letters 94, 081108 (2009); doi: $10.1063 / 1.3089359$

17. Attila Hilt, Gábor Járó, Attila Zólomy, Béatrice Cabon, Tibor Berceli, Tamás Marozsák: "Microwave Characterization of High-Speed pin Photodiodes", Proc. of the 9th Conference on Microwave Techniques COMITE’97, pp.21-24, Pardubice, Czech Republic, 16-17 Oct. 1997.

18. Stefan Lischke, Dieter Knoll, Christian Mai, Lars Zimmermann, Anna Peczek, Marcel Kroh, Andreas Trusch, Edgar Krune, Karsten Voigt, and A. Mai; High bandwidth, high responsivity waveguidecoupled germanium p-i-n photodiode ; Optics Express ; Vol. 23, Issue 21, pp.27213-27220 , (2015).

19. H. H. Li. Refractive index of silicon and germanium and its wavelength and temperature derivatives, $J$. Phys. Chem. Ref. Data 9, 561-658 (1993)

20. https://refractiveindex.info/?shelf=main\&book=Si\&page=Aspnes (Refractive index info) 